\title{
Hypophosphatasia: Rare but there
}

\author{
${ }^{1}$ Vikas K Jhingala, ${ }^{2}$ Pradeep Tangade, ${ }^{3}$ Himanshu Punia, ${ }^{4}$ Vipul Gupta, ${ }^{5}$ Vikas Singh \\ ${ }^{6}$ Monika Kalra, ${ }^{7}$ Suyash Chaudhary
}

\begin{abstract}
Hypophosphatasia (HP) is an inherited disorder characterized by defective bone and teeth mineralization and deficiency of serum and bone alkaline phosphatase (AP) activity. For severe forms, disease frequency has been estimated at 1/100,000. Symptoms of the disease are highly variable in their clinical expression ranging from stillbirth with unmineralized bone to early loss of tooth without any bone symptoms. The transmission of severe form is autosomal recessive while milder forms get transmitted as dominant or recessive autosomal traits. The diagnosis can be done by performing serum AP assay and molecular analysis of the tissue-nonspecific alkaline phosphatase (TNSALP) gene.
\end{abstract}

Keywords: Alkaline phosphate, Disorder, Recessive.

How to cite this article: Jhingala VK, Tangade $\mathrm{P}$, Punia $\mathrm{H}$, Gupta V, Singh V, Kalra M, Chaudhary S. Hypophosphatasia: Rare but there. J Contemp Dent 2016;6(3):184-188.

\section{Source of support: Nil}

Conflict of interest: None

\section{INTRODUCTION}

Hypophosphatasia (HP) is a rare metabolic bone disease characterized by suppressed activity of tissue-nonspecific alkaline phosphatase (TNSALP). This deficient activity of TNSALP has been attributed to mutations in TNSALP gene leading to significant alteration in the structure and functions of TNSALP. ${ }^{1}$

Until now, more than 190 different mutations have been recognized causing deficiency of TNSALP. Diagnosis of the disease is often complicated due to its rareness. Symptoms vary a lot in their clinical expression and can resemble other skeletal diseases. Due to its predominant autosomal recessive inheritance, defective genes can be passed in families unidentified for a long time; genetic studies showed a strong genotype - phenotype correlation, which means that patients with mild HP carry at least single mutation of significant residual enzymatic

${ }_{1,6,7}$ Postgraduate Student, ${ }^{2} \mathrm{Head},{ }^{3,4}$ Reader, ${ }^{5}$ Senior Lecturer

${ }^{1-7}$ Department of Public Health Dentistry, Teerthanker Mahaveer Dental College and Research Centre, Moradabad, Uttar Pradesh, India

Corresponding Author: Vikas K Jhingala, Postgraduate Student, Department of Public Health Dentistry, Teerthanker Mahaveer Dental College and Research Centre, Moradabad Uttar Pradesh, India, e-mail: jinghalavikas14@gmail.com activity, while patients with severe HP carry complete mutations leading to nil production of residual enzymatic activity. $^{2}$ The symptoms are highly variable in their clinical expression, ranging from stillbirth without mineralized bone to early loss of teeth without bone symptoms. ${ }^{3}$

\section{HISTORY}

The term "HP" was first used by Rathbun in 1948 when he described an infant boy who died from a severe form of rickets, weight loss, and fitting with a low level of the $\mathrm{L} / \mathrm{B} / \mathrm{K}$ isoenzyme of alkaline phosphatase (AP). The clinical sign of premature primary tooth loss was reported and the term "odontohypophosphatasia" (OHP) has been used for this phenomenon, implying incorrectly, considering it a different condition from true HP. In 1957, Fraser classified HP into three groups based on the age of presentation. ${ }^{4}$

\section{DISEASE NAME AND SYNONYMS}

Hypophosphatasia, Rathbun disease, phosphoethanolaminuria. $^{3}$

\section{CLINICAL CLASSIFICATION}

Four groups are described depending on the severity of skeletal disease, which, in turn, relates to the age at presentation. $^{4}$

\section{Perinatal HP}

- Usually present in utero.

- Stillbirth is a common finding while infants may live for a few days.

- Death commonly occurs due to an increased respiratory failure resulting from a rachitic chest.

- A complete lack of skeletal mineralization with shortened limbs and unusual spurs in the long bones is reported.

- Failure to gain weight, periodic apnea with bradycardia and cyanosis, cranial seizures and intracranial hemorrhage are some other features.

Radiographic features are diagnostic and take in irregular extensions of growth plate cartilages and unmineralized osteoid extending into the metaphyseal regions. The cranial bones are only ossified at the center and other membrane bones demonstrate a dominance of osteoid. 


\section{Infantile HP}

- Presents with lack of weight gain usually within the first 6 months of life due to poor feeding.

- There is often flail chest, blue sclera, and pneumonia.

- Hypercalcemia and hypercalciuria cause renal compromise and vomiting due to nephrocalcinosis.

- Cranial sutures remain wide with hypotonia and wide fontanelles.

- A raised intracranial pressure and hypertelorism may lead to proptosis.

Radiological findings are similar to the lethal form but less severe.

\section{Childhood HP}

- This form most commonly presents to the dental surgeon

- It has a very variable clinical picture, as does the adult form.

- Deciduous tooth loss is generally reported as a result of aplasia or cementum hypoplasia.

- Horizontal bone loss with no evidence of root resorption.

Radiographs of long bones may disclose "tongue-shaped" radiolucencies projecting from the epiphyseal growth plate into the metaphysis. Common features include widely open fontanelles with premature fusion of erania, rise in intracranial pressure, sutures often leading to proptosis, and brain damage. The calvarium has a "beaten copper" appearance resembling the "punched-out" lesions of multiple myeloma with reduced radiolucency.

\section{Adult HP}

- Diagnosed during middle age with a history of early deciduous or adult tooth loss.

- Anterior six maxillary and mandibular teeth are generally affected by premature tooth loss.

- Widespread caries of the adult dentition has been described.

- Liver metabolism may be affected as well as that of bone.

- Foot and ankle pain is common from metatarsal stress fractures, and this may also involve the thighs or hips due to femoral pseudofractures due to poor mineralization.

- The former appears first in childhood, enters a quiescent period, and then reappears in adulthood.

- The monophasic type is of later onset with a milder course.

Two other important terms are:

\section{Odontohypophosphatasia}

- The lower deciduous anterior teeth are most prone to exfoliation, but their roots remain entirely intact.
- The main lesion involves a quantitative and qualitative defect of cementum formation, although root resorption does sometimes occur.

- Commonly occurring features include delayed appearance of the deciduous dentition, enamel hypoplasia, and premature decay of the deciduous and adult dentitions, leading to total tooth loss before 20 years of age.

- No sex preference is reported, and mean age for the first tooth to be lost is 21 and 22 months.

Dentally, enlarged pulp spaces are reported as is reduced bone thickness of the mandibular angle. ${ }^{5}$

\section{Pseudo HP}

- Resembles classic HP with normal serum AP.

- There is osteopathy of long bones and skull, premature loss of deciduous teeth, hypercalcemia, hypotonia, and phosphoethanolaminuria. ${ }^{1}$

\section{ETIOLOGY}

Mutations of the liver/bone/kidney AP gene encoding TNAP or TNSALP is a major cause of this disease. The TNAP is generally a phosphomonoesterase of 507 residues, anchored at its carboxyl terminus to the plasma membrane by a phosphatidylinositol - glycan moiety. The enzyme is physiologically active in its dimeric form and serves extracellular substrates pyridoxal 5'-phosphate (PLP), phosphoethanolamine (PEA), and inorganic pyrophosphates (PPi). Its exact role in bone and dental mineralization is still unclear, but is caused by activities like hydrolysis of PPi, and mammalian-specific activities like collagen and calcium binding. The TNAP gene is located on chromosome 1p36.1, which consists of almost 12 exons distributed over $50 \mathrm{~kb}$. The gene is subject to high allelic heterogeneity, and more than 190 distinct mutations have been described. Most of them (79\%) are missense mutations. These mutations results in

- Highly variable clinical expressivity

- A great number of compound heterozygous genotypes.

\section{Genotype - Phenotype Correlations}

To assess the relative importance of these mutations, the genotype-phenotype relationship was performed on the basis of clinical data from patients, transfection studies, computer-assisted modeling, and studies of the biochemical properties of AP in cultured fibroblasts of patients or transfected cells. These experiments pave the way to the study of cell localization, cell degradation, and AP activity of mutant proteins. A good relation was seen between in vitro enzymatic activity of the mutant protein and disease severity. Patients with mild HP carry at least one 
mutation, which on testing exhibits significant residual enzymatic activity, whereas patients with severe HP carry mutations that, when tested, generally do not produce enzymatic activity. By using immunofluorescence and biochemical treatments, various mutations were observed and characterized for cell localization and degradation. ${ }^{3}$

\section{DENTAL ASPECTS}

Premature loss of primary teeth is allied with infantile, childhood, and OHP subtypes. Fraser reported that 75\% of the children in whom skeletal manifestations became apparent after 6 months of age experienced premature loss of primary teeth. The premature loss of teeth results from agenesis or hypoplasia of cementum, so that the exfoliated teeth are lost before resorption occurs. Mostly, the primary incisor teeth are the only teeth affected, and there are no associated inflammatory periodontal changes or root resorption. In the more severe forms of HP, posterior teeth may also exfoliate prematurely. Enamel hypoplasia, bulbous crowns, delayed dentin formation, and delayed eruption have also been reported. Although some have reported severe dental caries in association with this, it may be an incidental finding. The permanent dentition may be similarly affected.

Radiographic findings include enlarged pulp chambers and root canals with reduced alveolar bone. Histological analysis of exfoliated or extracted teeth reveals aplastic or hypoplastic cementum. As the only clinical manifestations in OHP are dental, the biochemical findings may be indistinguishable from mild forms of HP. ${ }^{6}$

\section{Role of Dental Cementum}

The role of cementum in the pathogenesis of the periodontal disease has received little attention. The cementum defects associated with HP have provided a unique model for the study of this periodontal tissue in relation to the pathogenesis of the other periodontal diseases. Page and Baarb reviewed the literature on early-onset periodontal conditions, in an effort to clarify the work of Gottlieb in which he suggested that it was an abnormality of cementum or "cementopathia" that underlies human periodontitis. They concluded that besides bacteria and host defense factors, root cementum defects may play a major role in determining the site specificity and susceptibility to early-onset periodontitis.

In a study by tight microscopy and transmitting and scanning electron microscopy of a single permanent incisor affected by HP, El-Labban et al suggested that the cementum defects in HP were unlikely to be due to defects of formation. The authors felt that since the resorption lacunae examined were filled with bacterial plaque and that there was evidence of cementum repair, the bacterial microflora must play a major role in the development of the cementum defects in HP. Baarb et al suggested that the bacterial destruction of the periodontal attachment apparatus in HP was the same basic process involved in adult periodontitis. Finally, there is renewed interest in the role of cementum in the pathogenesis and regeneration of the periodontium, with the identification of a novel cementum growth factor (Yonemura and Narayanan). Clearly, the HP model may be valuable in future assessment of such growth factors and their role in periodontal regeneration as well as destruction. ${ }^{5}$

\section{DIAGNOSTIC METHODS}

After clinical and radiographic examinations, HP diagnosis is based on laboratory assays and molecular biology, which appears to be very effective.

\section{Laboratory Assays}

Total serum AP activity is reduced in HP. So, the diagnosis can be suggested in individuals with serum clearly and consistently subnormal AP activity. In general, the severe the disease is, the lower the serum AP activity level appropriate for age. However, AP activity is only a helpful diagnostic indicator as other conditions may also show this finding, i.e., early pregnancy, hypothyroidism, anemia, drug administration, celiac disease, etc. It must be also noticed that serum AP dramatically varies with sex and age.

Increased urinary PEA levels support a diagnosis of $\mathrm{HP}$, but is not pathognomonic. It is also seen in a variety of other conditions, including several metabolic bone diseases, and some of the HP patients may show normal PEA excretion. Hence, the demonstration that PEA is also a natural substrate of TNAP in vivo remains to be confirmed.

Increased PLP may be a sensitive marker for HP.

Heterozygous carriers of the severe forms are usually normal clinically, but often show reduced serum AP activity and increased urinary PEA.

\section{Molecular Biology}

Screening for mutations in the TNAP gene is essential to confirm the HP diagnosis when biochemical and clinical data are not clear enough, to offer genetic counseling or molecular prenatal diagnosis to families affected by severe forms of the disease. Clinical and biochemical data may not always distinguish HP from other skeletal diseases, such as osteogenesis imperfecta. Mutation screening can be performed by single-stranded conformation polymorphism (SSCP) or denaturing gradient gel electrophoresis (DGGE) followed by sequencing of exons exhibiting variants, by direct sequencing of the complementary deoxyribonucleic acid (cDNA) or by direct sequencing of genomic 
sequences. The exons are small and few in number, so can be easily analyzed. However, the mutations are spread all over the exons, so the whole coding sequence has to be analyzed. In addition, some mutations remain undetectable due to exhaustive sequencing of the coding sequence, intron-exon borders, and untranslated exons. This may be due to mutations lying in intronic or regulatory sequences or due to the expression of heterozygous mutations, especially in moderate (childhood, adult, and odonto) HP. By using sequencing, approximately $95 \%$ of mutations are detected in severe (perinatal and infantile) $\mathrm{HP}$, whereas patients with mild forms often carry only one detected mutated allele. ${ }^{3}$

\section{MANAGEMENT AND TREATMENT}

At the moment, there is no curative treatment for HP. Therefore, symptomatic treatment in order to improve the clinical features, especially with regard to seizures, pain, and other metabolic phenomena (e.g., nephrocalcinosis) is of major interest. We have previously described the presence of hyperprostaglandinism in childhood HP.

An anti-inflammatory treatment using nonsteroidal anti-inflammatory drugs (NSAIDs) resulted in a significant improvement of physical activity, reduction in pain, and normalization of prostaglandin (PG) levels. After drug withdrawal, the positive effect persisted for another 3 to 6 weeks. The NSAID treatment was safe and should be considered in patients with complaints of pain.

Treatment with magnesium and zinc, the catalytic ions of the enzyme, and PLP has no significant long-term benefit for HP patients.

Dietary phosphate restriction is recommended for patients with high serum levels of phosphate. Especially for children with persistent growth retardation, an adequate nutrition with the support of a professional dietician is of major importance.

Supplementation with vitamin $\mathrm{D}$ can be recommended according to the guidelines for healthy children. Further, additional vitamin D therapy, as it is used in vitamin D-deficient rickets, should be avoided. An additional supplementation of calcium may result in hypercalcemia and formation of kidney stones. Treatment strategies should be monitored permanently in a HP-experienced clinic. ${ }^{2}$

Different approaches have been used to improve the condition of severe cases of HP where bony abnormality is of major concern. These approaches include a supplement of vitamin $\mathrm{D}$, phosphate, or combination of vitamin D and fluoride (Rasmussen 1983). ${ }^{7}$

Physical exercises like cycling and swimming as well as physiotherapy seem to be useful tools for muscle building in order to stabilize the skeleton. Whether a physical workout leads to appositional growth of bone in HP has not been evaluated so far. The maintenance of physical function is of major importance. Especially in severe forms of disease, trauma to the skeleton must be avoided because resulting fractures take a long time to heal and might be difficult to treat. Surgical as well as conservative therapeutic approaches should be discussed with a HP-experienced clinical center. Premature fusion of cranial sutures may result in secondary ectopia of the cerebellar tonsils or formation of hydrosyringomyelia.

Neurosurgical interventions are necessary in patients with neurologic symptoms like permanent headache, seizures, paralysis, or numbness of extremities as well as papilledema due to elevated intracranial pressure. With regard to the progressive nature of craniosynostosis, careful surveillance including ophthalmoscopic reassessment, neurologic examination, and radiographic imaging is recommended throughout childhood until adolescence and in presence of Chiari I malformation or hydrosyringomyelia, probably throughout lifetime.

Enzyme replacement therapy (ERT) and allogeneic stem cell transplantation are causative and potentially curative attempts of treatment. The ERT is still in development. First results using a knockout mouse model were promising and supported the initiation of a phase I clinical trial in the United States and Canada. There is only one casuistic report of haploidentical bone marrow transplantation in a patient with very severe HP. Initial response was reported favorable; however, the long-term outcome has not been reported. ${ }^{2}$

Dental monitoring by a HP-experienced dentist or orthodontist seems to be very important. ${ }^{2}$

In mild types of the condition where the primary teeth are exfoliating prematurely, the major concern is to maintain the arch length until the permanent dentition erupts.?

Prosthetic replacement of prematurely lost teeth may be helpful for linguistic development and social integration of patients in preschool or school. Possible loosening of remaining teeth should be noted in planning removable dentures. Periodical dental supervisions are essential. Impaired transversal development (width of jaw) of front teeth in the upper and lower jaw due to premature exfoliation of primary teeth may result in a nonalignment of permanent front teeth.

Corrections of such incorrect teeth positions are possible, but should be coordinated by a HP-experienced orthodontist.

Grewal and Gupta ${ }^{8}$ treated a case with prosthetic rehabilitation of a 16-year-old female patient with anodontia and no occlusion. Patient was managed clinically 
by saving her rest of the teeth, a maxillary overdenture, and mandibular cast partial denture.

\section{CONCLUSION}

It is a rare metabolic disorder due to genetic variations. Milder cases are more common. Dental aspect includes premature loss of primary teeth. Pediatric dentist should diagnose the type of cases when an uncommon case of primary tooth exfoliation is encountered. Various treatment options can be chosen for treatment of this disorder. Prevention of shortening of dental arches is a major issue until permanent teeth erupt. Oral hygiene maintenance and prosthetic replacement can be a choice of treatment.

\section{REFERENCES}

1. Manohar B, Mathur L, Pillai R, Shetty N, Bhatia A, Mathur A. Odontohypophosphatasia - a case report. J Carcinogene Mutagene 2010;1(2):105.
2. Beck C, Morbach H, Stenzel M, Collmann H, Schneider P, Girschick HJ. Hypophosphatasia - recent advances in diagnosis and treatment. Open Bone J 2009;1:8-15.

3. Mornet E. Hypophosphatasia. Orphanet J Rare Dis 2007 Oct;2:40.

4. Chapple IL, Thorpe GH, Smith JM, Saxby MS, Glenwright HD, Green A, Perry GM, Grundy M, Shaw L, Matthews JB. Hypophosphatasia: a family study involving a case diagnosed from gingival crevicular fluid. J Oral Pathol Med 1992 Oct;21(9):426-431.

5. Chapple ILC. Hypophosphatasia: dental aspects and mode of inheritance. J Clin Periodontol 1993 Oct;20(9):615-622.

6. Hollis A, Arundel P, High A, Balmer R. Current concepts in hypophosphatasia: case report and literature review. Int J Paediatr Dent 2013 May;23(3):153-159.

7. Cheung WS. A mild form of hypophosphatasia as a cause of premature exfoliation of primary teeth: report of two cases. Pediatr Dent 1987 Mar;9(1):49-52.

8. Grewal PS, Gupta KP. Prosthetic rehabilitation of a young patient with hypophosphatasia - a review and case report. Contemp Clin Dent 2012 Jan;3(1):74-77. 